\title{
Insights from neuropsychology: pinpointing the role of the posterior parietal cortex in episodic and working memory
}

\section{Marian E. Berryhill *}

Department of Psychology, Program in Cognitive and Brain Sciences, University of Nevada, Reno, NV, USA

Edited by:

Michael Rugg, University of Texas at Dallas, USA

\section{Reviewed by:}

Patrizia Fattori, University of

Bologna, Italy

Melina Uncapher, Stanford

University, USA

${ }^{*}$ Correspondence:

Marian E. Berryhill, Department of Psychology, Program in Cognitive and Brain Sciences, University of Nevada, Mail Stop 296, Reno, NV 89557, USA

e-mail: mberryhill@unr.edu
The role of posterior parietal cortex (PPC) in various forms of memory is a current topic of interest in the broader field of cognitive neuroscience. This large cortical region has been linked with a wide range of mnemonic functions affecting each stage of memory processing: encoding, maintenance, and retrieval. Yet, the precise role of the PPC in memory remains mysterious and controversial. Progress in understanding PPC function will require researchers to incorporate findings in a convergent manner from multiple experimental techniques rather than emphasizing a particular type of data. To facilitate this process, here, we review findings from the human neuropsychological research and examine the consequences to memory following PPC damage. Recent patient-based research findings have investigated two typically disconnected fields: working memory (WM) and episodic memory. The findings from patient participants with unilateral and bilateral PPC lesions performing diverse experimental paradigms are summarized. These findings are then related to findings from other techniques including neurostimulation (TMS and tDCS) and the influential and more abundant functional neuroimaging literature. We then review the strengths and weaknesses of hypotheses proposed to account for PPC function in these forms of memory. Finally, we address what missing evidence is needed to clarify the role(s) of the PPC in memory.

Keywords: parietal lobe, parietal lesion, working memory, episodic memory, autobiographical memory, short-term memory, neuropsychology, source memory
Several years ago the posterior parietal cortex (PPC) stepped into the spotlight as an area of interest in cognitive neuroscience. Traditionally, vague terms such as "association cortex" were used to describe the multiple functions of the PPC. Association cortex referred to the intermediate stage of processing in between primary sensory cortices and frontal areas involved in executive function. The PPC covers a large territory and includes a number of distinct cortical regions including the superior parietal lobule (SPL, BA 7) and the inferior parietal lobule (IPL, BA 39, 40), which includes the angular and supramarginal gyri. Apart from these gross distinctions others propose more detailed parietal parcellations based on analyses of functional and structural connectivity (e.g., Nelson et al., 2010; Uddin et al., 2010; Caspers et al., 2012). Portions of the PPC have been associated with a wide-ranging array of cognitive roles including spatial or selective attention (especially right IPL, reviewed in Chambers and Mattingley, 2005; Husain and Nachev, 2007; Driver et al., 2010; Corbetta and Shulman, 2011), stimulus selection (reviewed in Corbetta and Shulman, 2002), navigation (reviewed in Berthoz, 1997; Postma et al., 2008), visual perception (especially left SPL, IPL: reviewed in Landis, 2000; Berman and Colby, 2009; Pisella et al., 2009), action planning and control (anterior intraparietal sulcus, IPL, SPL; reviewed in Glover, 2004; Tunik et al., 2007; Jax and Coslett, 2009), tool use (IPL: reviewed in Frey, 2008; Arbib et al., 2009), reorienting (IPL: reviewed in Maurizio Corbetta et al., 2008), executive function (SPL, IPL: for a meta-analysis see Niendam et al., 2012), and even general intelligence (SPL, IPL: Gläscher et al., 2010). Clearly, regions of the PPC were considered important for many aspects of cognition, but it had not been linked to memory.

The era of functional magnetic neuroimaging (fMRI) created an opportunity to revisit assumptions regarding brain structurefunction links. Of relevance here was the unexpected number of reports in the episodic memory literature that identified PPC activations during memory tasks. In a seminal review paper, Wagner and colleagues reviewed these fMRI findings and presented three plausible roles for PPC involvement in episodic memory (Wagner et al., 2005). The three proposals attention to internal representations, output buffer, and mnemonic accumulator are discussed below. This paper prompted a surge of interest in the PPC/memory relationship as evidenced by $350+$ citations since publication.

Thus, research proposing PPC involvement in memory is relatively recent and for many it comes with a large grain of salt. This is in part because neuropsychological patients with PPC damage do not have predominant memory deficits. Instead these patients present with primarily spatial and attentional problems. The complete list of symptoms associated with PPC lesions is highly varied. Damage to the left IPL (angular gyrus) can lead to Gerstmann's syndrome (finger agnosia, left-right confusion, 
dyscalculia, dysgraphia), whereas right IPL damage often produces hemispatial neglect, and bilateral IPL damage can result in Balint's syndrome (optic ataxia, optic apraxia, simultanagnosia). It is important to acknowledge one of the caveats of neuropsychological studies is that the lesions themselves do not obey the boundaries between different functional regions within the PPC. This is largely due to the broad vascular territory of the middle cerebral artery over the lateral PPC. Infarcts of the middle cerebral artery reliably damage multiple regions in the PPC. Furthermore, even small lesions may cause damage to neighboring structures in and connected to the lesion site. Therefore it is difficult to find patients with damage isolated to structures solely within the IPL or the SPL. It is also true that researchers do not always have access to clear brain scans and they may not be able to report detailed lesion locations. Consequently, because of this lack of specificity, the more general term PPC is used with regard to patients' lesions unless otherwise noted.

Caveats aside, recent findings suggesting that the predominant symptoms occurring after PPC damage might obscure nuanced memory deficits. This has prompted researchers to revisit this question. Below, I briefly review neuropsychological research investigating episodic memory performance in people with PPC lesions; see Table 1. The subsequent section will summarize investigations of working memory (WM). Although episodic and WM are generally addressed separately, they are both included here because both literatures are relatively small, and often patient participants are tested in both domains. More importantly, there is evidence to suggest that both episodic and WM cross-contaminate tasks devoted ostensibly to one or the other (Cowan, 2008). In other words, you often get both episodic and WM contributing to task performance.

This paper updates an earlier review on a similar topic (Olson and Berryhill, 2009) and complements reviews considering PPC activations in episodic memory tasks (Wagner et al., 2005; Rugg and Curran, 2007; Cabeza, 2008; Cabeza et al., 2008; Vilberg and Rugg, 2008; Hutchinson et al., 2009; Uncapher and Wagner, 2009; Shimamura, 2011) and the conflict between the MRI and patient literatures (Schoo et al., 2011).

\section{NEUROIMAGING IDENTIFIES PPC ACTIVATIONS DURING EPISODIC MEMORY}

This brief paragraph serves to highlight several observations from neuroimaging data that prompted neuropsychological investigations of episodic memory in people with PPC lesions. Neuroimaging studies first noted that memory tasks reliably elicited PPC activations (e.g., Wojciulik and Kanwisher, 1999; Culham and Kanwisher, 2001). One characteristic pattern of PPC activation in memory studies is known as the parietal old/new effect. This refers to increased activity in the lateral PPC when a participant performing a recognition memory test endorses a stimulus as previously encountered ("old") compared to when the participant rejects a stimulus as novel ("new"), even if their response was incorrect (Kahn et al., 2004; Wheeler and Buckner, 2004; Rugg and Curran, 2007; Kim, 2011). The parietal old/new effect is complemented by a second confidencerelated pattern in the PPC. Two dissociable regions respond more robustly to high (inferior IPL) or low (superior IPL) response confidence, again regardless of response accuracy (Kim and Cabeza, 2007, 2009). In both examples, the neural activity in the PPC corresponds to the participant's subjective experience of memory. Other PPC activations reflect response accuracy such that the area in and around the intraparietal sulcus is activated more strongly by correctly remembered items than correctly rejected items (Wagner et al., 2005). Finally, overlapping medial PPC (precuneus) activations are reported during assorted episodic memory tasks tapping associative memory, autobiographical memory and episodic future thinking (reviewed in Addis et al., 2007; Buckner and Carroll, 2007; Cabeza and St. Jacques, 2007; Burgess et al., 2011; for recent findings see also: Burianova et al., 2010; St. Jacques et al., 2011; Addis et al., 2012).

\section{EPISODIC MEMORY AFTER PPC LESIONS}

Traditionally, clinicians do not associate memory loss with parietal damage. Indeed, one of the classic texts on PPC function has chapters on visual, tactile, spatial, language, and body-image deficits (Critchley, 1953). Indeed, the well-known British neurologist MacDonald Critchley referred to memory deficits after PPC damage exclusively in terms of impaired spatial imagery (Critchley, 1953). However, there was some indication that patients with parietal damage and hemispatial neglect could have visual (Butters et al., 1970) or auditory memory deficits (Heilman et al., 1974) for stimuli presented in the neglected field contralateral to the lesion. In these studies, stimuli were presented to either the intact or neglected hemifield and after a delay, memory performance was tested. Performance for stimuli presented in the neglected field was worse than for stimuli in the intact field. Verbal WM deficits were also associated with nonspecific left hemisphere damage in patients with aphasia (Warrington et al., 1971; De Renzi and Nichelli, 1975). One difficulty with these earlier findings is the perceptual confound-if a stimulus was not attended due to neglect, performance in memory tasks would also be impaired. Secondly, in many patient-based studies there was a lack of anatomical specificity to describe the location of lesions. Consequently, it remained unclear whether there were any subtle memory deficits associated with PPC damage, per se. The increased use of neuroimaging has largely solved the problem of precisely defining the lesion location.

In one more recent case implicating the PPC in episodic memory a closed head injury caused damage to bilateral occipital and the right PPC in a 19-year-old man, patient D.H. (Hunkin et al., 1995). In spite of high-normal intelligence, patient D.H. was able to spontaneously remember only one autobiographical event occurring prior to the accident. Other events had been retold to him, but he claimed that they were not accompanied by a sense that of having personally experienced the events. Semantic information detailing his youth had been relearned and was normal. D.H. did not have anterograde deficits, meaning he had no difficulty retrieving autobiographical events occurring after his accident. In short, his memory deficit was limited to autobiographical events before his brain damage. Deficits in autobiographical memory have been found in several other studies. Davidson and colleagues report the case of patient S.M. who had surgery to remove a tumor in the left parietal lobe 
Table 1 | Summary of neuropsychological investigations of non-spatial episodic and working memory.

\begin{tabular}{|c|c|c|c|c|}
\hline Authors & Lesion location & Type of memory & Test & Memory deficits \\
\hline 2. Berryhill et al., 2010a & $\mathrm{Bi} \mathrm{PPC}^{*}$ & $\begin{array}{l}\text { Episodic future } \\
\text { thinking }\end{array}$ & Imagine future & Fewer episodic details \\
\hline 3. Davidson et al., 2008 & L PPC (lateral, IPL, SPL) & Autobio LTM & Autobio; DRM & $\begin{array}{l}\text { Fewer details; Impaired } \\
\text { recollection and subjective } \\
\text { experience }\end{array}$ \\
\hline 4. Drowos et al., 2010 & $\mathrm{Bi} P P C^{*}$ & LTM & Visual/verbal DRM & Fewer false alarms \\
\hline 5. Vuilleumier et al., 2002 & $\begin{array}{l}\text { R PPC w/neglect (mixed } \\
\text { cortical and white matter) }\end{array}$ & LTM & Object & $\begin{array}{l}\text { Impaired explicit memory for } \\
\text { contralaterally presented and } \\
\text { extinguished items }\end{array}$ \\
\hline 9. Berryhill et al., 2011 & $B i P P C^{*}$ & WM/LTM & Recognition/Recall & $\begin{array}{l}\text { Recognition when blocked; } \\
\text { Recall when rehearsal was } \\
\text { prevented }\end{array}$ \\
\hline \multicolumn{5}{|c|}{ PPC PATIENT STUDIES SHOWING INTACT MEMORY } \\
\hline 10. Milner, 1968 & Uni PPC (L, R IPL, SPL) & LTM & Recognition memory & Intact face/pattern memory \\
\hline 11. Satoh et al., 2011 & $R P P C(I P L)$ & LTM & $\begin{array}{l}\text { Daily memory (Rivermead), } \\
\text { Verbal memory }\end{array}$ & Musical anhedonia \\
\hline 12. Simons et al., 2008 & Uni PPC $(L, R, I P L, S P L)$ & LTM & Source memory & - \\
\hline
\end{tabular}

For a table of spatial memory deficits please see Olson and Berryhill (2009).

Entries in italics indicate null findings with regard to memory or WM deficits.

Abbreviations: Bi PPC, bilateral posterior parietal cortex; R, right; L, left; DRM, Deese-Roedigger-McDermott (Deese, 1959; Roediger and McDermott, 1995); Autobio, autobiographical memory; LTM, long term memory; AMI, autobiographical memory interview (Kopelman et al., 1989); Levine, Levine autobiographical memory interview (Levine, 2004); *, see Row 1.

(Davidson et al., 2008). S.M. volunteered that her autobiographical memories did not seem to be accompanied by a sense of having experienced the event herself. However, her memory impairment was broader than that observed in D.H. She was also impaired on the paired-associates subtest of the Wechsler Memory Scale (Wechsler, 1997) and she had low confidence in other memory tasks.

We were able to test two rare patients with bilateral parietal damage in a series of episodic and WM studies, including tests of autobiographical memory. The primary deficit associated with bilateral parietal lesions is simultanagnosia, a component of Balint's syndrome (Balint, 1909). Simultanagnosia is the inability to attend to more than one object at a time. In other words, patients are only able to report the presence of a single item at any moment. Not surprisingly, this piecemeal visual experience renders global percepts of visual scenes impossible. Although both patients had bilateral damage, their lesions were largely non-overlapping. TQ591 had both bilateral IPL and right SPL damage as well as left precuneus involvement and EE555 had more lateral and inferior lesions in the IPL. We administered two autobiographical memory tests (Kopelman et al., 1989; Levine et al., 2002). The first test (Kopelman et al., 1989) emphasized semantic knowledge for different epochs in time, and here the PPC patients performed relatively normally. In the second test (Levine et al., 2002) participants freely recalled events from several time points. These descriptions were followed by a series of specific probe questions to elicit additional details related to each retrieved memory. The two bilateral PPC patients, EE555 and TQ591, were able to freely recall memories, but they were impoverished and significantly lacked details when compared to control participants. However, they performed normally when specific probe questions were provided. This pattern of deficits revealed a dissociation between spontaneous and guided memory retrieval that was inconsistent with a global memory impairment. These data demonstrated that PPC damage impaired patients' abilities to spontaneously and vividly retrieve memories, but absent details could be accessed when memory retrieval cues provided support (Berryhill et al., 2007).

We later tested, whether these two bilateral PPC patients were impaired at describing future events. This type of task is 
referred to as "episodic future thinking," or "constructed experience" and it is thought to tap episodic memory retrieval to anticipate the outcome of events yet to happen. Neuroimaging studies of episodic future thinking and autobiographical memory identify overlapping activity in frontoparietal networks (reviewed in Addis et al., 2007; Buckner and Carroll, 2007; Hassabis and Maguire, 2007). We used a paradigm similar to the autobiographical memory paradigm described above (Levine et al., 2002). Verbal prompts cued participants to describe future events (e.g., "Imagine you are meeting a friend for lunch") replicating an approach used with amnesic patients (Hassabis et al., 2007). Performance was measured using text analyses that tallied the numbers of freely reported details. There were also measures of subjective experience in which participants were asked to rate how present and salient the imagined events felt and the overall quality of the conjured experienced. Our results showed that the two bilateral PPC patients were impaired in their ability to envision richly detailed future events (Berryhill et al., 2010a). More precisely, the bilateral PPC patients' responses were significantly lower than those of controls for measures of spatial integration and overall quality. Somewhat surprisingly, given previous indications that PPC lesions can reduce a sense of subjective experience, the bilateral PPC patients did not rate their sense of presence or salience significantly lower than healthy controls. Here, again, was evidence of a restricted deficit in episodic memory. Constructed experience has been evaluated in at least one other patient group with parietal-area damage. Patient participants with diffuse axonal injury were tested on constructed memory and this performance was correlated with damaged areas as identified through diffusion tensor imaging (Kondo et al., 2010). In these patients PPC damage (inferior IPL) correlated with performance on the constructed memory task (Kondo et al., 2010).

The autobiographical and constructed experience findings from the patients with bilateral PPC lesions used similar free recall paradigms with similar advantages. One advantage was to minimize encoding demands since the events occurred prior to the lesions or could be assembled collage-style from premorbid occurrences. A second advantage of these studies was that the instructions were simple and judging from the length of transcripts, people enjoyed participating. Drawbacks of these studies are that scoring requiring a text analysis that is enormously time consuming and requires some level of rater subjectivity, and the bulk of the episodic memory field uses more standardized experimental paradigms. Below, we review the findings from more commonly used experimental paradigms. Two papers used the Deese-Roediger-McDermott (DRM) (Deese, 1959; Roediger and McDermott, 1995) false-memory paradigm to test patient participants with PPC damage (Davidson et al., 2008; Drowos et al., 2010). In the DRM paradigm, lists of semantically related items are presented (e.g., pillow, night, blanket). Next, participants perform a delayed memory recognition task in which they make old/new judgments and for old responses they make a remember/know judgment reflecting whether there was a clear recollection or a sense of familiarity for the stimulus. The essential finding is that there is a high false alarm rate to new lure items that are closely related to a stimulus list (e.g., sleep). The aforementioned patient S.M. performed abnormally, when compared to control participants by having lower recognition accuracy and reduced recollection as indicated by significantly fewer remember responses (Davidson et al., 2008). Her performance also reflected fewer false alarms to the lure words. We also tested patients EE555 and TQ591 using the visual and verbal versions of the DRM paradigm (Drowos et al., 2010). As with patient S.M., the bilateral PPC patients performed abnormally from control participants by committing fewer false alarms to lure words and by making fewer remember responses to the false alarms they did make. However, when we used visual stimuli instead of verbal stimuli, a different pattern emerged. Visual DRM studies do not tend to have the degree of false memories because of the "distinctiveness heuristic" rendering visually presented stimuli more distinctive (e.g., Israel and Schacter, 1997). If the PPC damage impaired gist perception due to a deficit in recollection, as proposed by Davidson et al. (2008) we should have seen the same pattern for auditory or visual versions of the DRM. The bilateral PPC patients performed more accurately in the visual DRM. However, EE555 was unable to take advantage of the distinctiveness heuristic and she maintained the same level of false memories across both versions of the DRM. EE555's remember/know responses support the idea that she was less confident in her memory, though, as she made only a few remember responses. It is also important to note that the bilateral PPC patients did not have a general problem with gist information. They performed normally when recounting the thematic memory for short stories, although again, they reported fewer details than control participants (Drowos et al., 2010).

Memory impairment for learning paired-associates has been tested in unilateral and bilateral PPC patient populations (Davidson et al., 2008; Berryhill et al., 2009). In a set of three paired-associates experiments testing the bilateral PPC patients, we found that they were not impaired on the memory retrieval aspect of a word pair task (Giovanello et al., 2006), linking variable amounts of information with items in a fan task (Radvansky, 2005), or at learning audio/visual pairs. In the word pairs and audio/visual pairs tasks response confidence was measured in two different ways: using the remember/know procedure and as a numeric (1-6) rating, respectively. The PPC patients' numeric confidence ratings were significantly lower than the control participants in the audio/visual task, but the number of remember responses was no different from controls in the word pairs experiment (Berryhill et al., 2009). We concluded that the PPC patients had reduced confidence in their responses.

Several researchers have conducted investigations looking at source memory, the ability to retrieve specific details of the encoding stage. For example, a source memory task may require you to remember whether you heard the news on the radio or on the television. There are several findings reporting normal source memory in patients with PPC damage (Ally et al., 2008; Simons et al., 2008, 2010). First, Simons and colleagues selected participants with unilateral parietal damage in areas that overlap with fMRI brain activations observed during source memory tasks (Simons et al., 2008; for relevant findings see also Duarte et al., 2011). At encoding, participants were shown words or pictures and for each item they were asked to judge whether the item was pleasant/unpleasant or from entertainment/politics. At test, participants had to remember which judgment they had made for 
each stimulus. The patients performed normally on the source memory judgment. Subsequently, Simons et al. (2010) conducted a series of three source memory tasks in unilateral and bilateral PPC patients (including bilateral PPC patients EE555 and TQ591). Here, participants heard sentences presented in a male or female voice. At test, they indicated whether the sentence was old or new, rated their confidence and then judged whether the original voice had been male or female and finally, how confident they were in their response. All participants and patients performed the old/new recognition task normally and had normal confidence ratings. There were no group differences in the source judgment task either. However, the bilateral PPC patients had significantly lower confidence in their source judgments. A follow-up experiment using visual stimuli found the same dissociation between normal source memory accuracy accompanied by impaired confidence. Davidson et al. (2008) found a similar dissociation in patients with unilateral PPC damage. He presented words with definitions in visual or auditory domains and later asked participants to retrieve the sensory domain at encoding and to make remember or know responses. While the patients performed accurately when performing the source memory judgment, they made significantly fewer remember responses. This is evidence that recollection confidence declines after PPC damage.

One last study tested both unilateral right and left PPC patients in a recognition memory paradigm. Haramati et al. (2008) presented participants with pictures, sounds, and words and asked for preference ratings at encoding. Later, participants performed a delayed recognition test. Only the right PPC patients had any deficits, and these were limited to poor recognition for pictures and sounds, but not words (Haramati et al., 2008). Tempering this finding were voxel-based analyses of lesion location and performance suggested that the overlap in right frontal regions, rather than parietal cortex, predicted memory deficits.

\section{ROLE OF THE PPC IN EPISODIC MEMORY}

The above findings present nuanced rather than global memory deficits. Where medial temporal lobe amnesics have dramatic memory impairments, PPC patients have modest deficits. The data described above has been used to support several different mechanisms of PPC involvement in episodic memory. Wagner et al. (2005) described three separate proposals, attention to internal representations, output buffer, mnemonic accumulator, that have been very helpful springboards for neuropsychological researchers. A fourth proposal is the cortical binding of relational activity (CoBRA) theory (Shimamura, 2011). Finally, Davidson et al. (2008) developed an alternative view, the subjective experience hypothesis. These views are discussed below.

\section{ATTENTION TO INTERNAL REPRESENTATIONS}

The initial proposal implicated the PPC in directing attention to the mental representation of a memory. A related elaboration, the attention to internal memory (AtoM: Cabeza, 2008; Cabeza et al., 2008) proposed a distinction between spontaneously retrieved memories and deliberately sought memories akin to the distinction between bottom-up and top-down attention. Here, the proposal identifies the role of the IPL is involved in bottom-up guided, spontaneous retrieval, whereas the SPL is responsible for top-down directed, effortful retrieval. This view accounts for findings in PPC patients with more inferior lesions who show normal performance when memory cues are provided (intact top-down retrieval) and impaired performance when they must retrieve the memory on their own (impaired bottom-up retrieval). This perspective has been criticized in a large fMRI meta-analysis that failed to reveal an inferior/superior subdivision based on whether the retrieval event was mediated by top-down or bottom-up attention (Hutchinson et al., 2009). However, at least one fMRI finding identifies IPL activations during bottomup retrieval of a paired associate and SPL activations during top-down retrieval. Furthermore, these neuroimaging findings extended to PPC patients with IPL and SPL lesions (Ciaramelli et al., 2010).

\section{EPISODIC OR OUTPUT BUFFER}

This explanation proposes that the PPC serves as one module within the multimodal model of memory called the episodic buffer (Baddeley, 2000). The episodic buffer is hypothesized to maintain sensory and mnemonic information in a common "language" or representation. It may serve as a sort of Esperanto for the brain. The information in the buffer is thus available for manipulation and is closely related to WM function. This view cannot account for the finding that associative memory for audio/visual pairs remains intact (Berryhill et al., 2009), because multimodal storage is necessary for these types of tasks. Secondly, this explanation predicts that PPC patients should have poor performance in tasks asking for narrative structure, such as the autobiographical or constructed experience tasks (Berryhill et al., 2007, 2010a). However, although lacking rich details, the patients provide accounts with intact and appropriate narrative structure.

\section{MNEMONIC ACCUMULATOR}

This proposal suggests that PPC neurons register an index signaling memory retrieval status and that when this index passes threshold, participants would endorse an item as old. In other words, this proposal endows the PPC with the ability to measure memory strength. A prediction of this hypothesis is that memory decision-making would be impaired after PPC damage. However, PPC patients do not have general memory deficits as would be predicted if they had difficulty interpreting a missing or damaged index of memory strength. Instead the deficits that emerge tend to relate to memory confidence even when memory accuracy is intact (Haramati et al., 2008; Berryhill et al., 2009; Drowos et al., 2010).

\section{CORTICAL BINDING OF RELATIONAL ACTIVITY (COBRA)}

Shimamura recently proposed that the ventral PPC interacts with the prefrontal cortex and medial temporal lobe and creates a cortical network of the details related to an episodic memory (Shimamura, 2011). According to CoBRA, medial temporal lobe regions form associations at encoding, but through consolidation the vPPC becomes more important for reactivating, or to use their term "re-collecting," the ensemble of relevant episodic details. CoBRA predicts that there should be greater vPPC activity when memories contain greater multimodal details because their retrieval requires more cross-cortical 
links of feature information. A second prediction is that the vPPC should be more involved when the retrieval process relies more on recollection than familiarity. A potential challenge for CoBRA is the finding that patients with PPC lesions perform normally at source memory tasks (Simons et al., 2008, 2010). However, Shimamura suggests that these types of source memory tasks may not be sufficiently multimodal to require vPPC activity. A second potential problem is that patients with bilateral parietal damage perform normally at associative learning tasks even those that require multimodal pairings (Berryhill et al., 2009).

\section{SUBJECTIVE EXPERIENCE}

This view puts forth the idea that the PPC signals the perceived oldness of an event. In essence, this distinguishes between something vividly retrieved versus something vaguely recalled. Proponents suggest that damage to this processing explains findings of reduced confidence in PPC patients' memories across various paradigms (Hunkin et al., 1995; Ally et al., 2008; Davidson et al., 2008; Berryhill et al., 2009; Drowos et al., 2010; Simons et al., 2010). However, reduced memory confidence is not universal and the subjective experience account does not currently predict why some memory tasks are accompanied by reduced memory and others are not.

Our own view has been to create a hybrid of two existing proposals: the subjective experience and attention to internal representations (Berryhill et al., 2009; Drowos et al., 2010). The advantage for this merger is that it includes a role for the PPC in strategically accessing and attending to the full set of details associated with a particular event. It also includes the function of assessing the vivid richness of memories to account for the deficits in memory confidence. In other words, when the PPC is damaged, patients may not be able to fully reactivate the full assembly of stored details to revivify the event. This would lead to impoverished recollections and a reduced sense of re-experiencing a past event.

\section{PPC AND WORKING MEMORY}

In cognition, WM is a core executive function that allows us to maintain information over short delays. Neurophysiological data provided the first indications that neurons in the PPC were involved in maintaining WM representations. Recordings from non-human primates indicated that PPC neurons, in addition to the prefrontal cortex, maintained their activity after during the delay period when there were no stimuli available (e.g., Chafee and Goldman-Rakic, 1998, reviewed in Constantinidis and Procyk, 2004; Rawley and Constantinidis, 2009; for work in rodents see review by Bucci, 2009).

It may strike some as disjointed to pair episodic memory with WM given the fact that these two topics reside in different literatures. Others make the case quite clearly that it is difficult to develop tasks that precisely test episodic or WM alone (Cowan, 2008). In disentangling the interplay of medial temporal, prefrontal, and PPC structures in various forms of memory, it is important to look at WM. The traditional division between episodic and WM was established by classic neuropsychological research in medial temporal lobe amnesia patients (e.g., Scoville and Milner, 1957). However, recent findings suggests that amnesics have abnormal WM performance even at short delay intervals (Warrington and Taylor, 1973; Ryan and Cohen, 2004; Ranganath and D'Esposito, 2005; Hannula et al., 2006; Olson et al., 2006a,b; Ezzyat and Olson, 2008; but see Baddeley et al., 2010). For example, medial temporal lobe amnesic patients were less accurate and slower when remembering a single face over a 1 s delay interval (Ezzyat and Olson, 2008). These findings point toward greater interactions between episodic memory and WM than was previously appreciated (see also: Ranganath et al., 2005; Jonides et al., 2008; Graham et al., 2010).

In addition, a series of high-impact findings in the fMRI literature indicated that PPC activations reflected the number of items maintained in WM (Todd and Marois, 2004, 2005; Xu and Chun, 2005). In these studies, the IPL, specifically the intraparietal sulcus, showed parametric increases in activity up to the behavioral WM capacity limit. In the ERP literature, a similar finding, the contralateral delay activity, also underscored the importance of posterior regions in WM maintenance (reviewed in Drew et al., 2006). The prediction from these neuroimaging data was clear: PPC damage should lead to WM deficits.

\section{PPC DAMAGE AND WM}

The majority of WM studies in neuropsychological patients with PPC lesions test performance in spatial WM tasks. This is due to the association of parietal cortex with spatial attention. Spatial WM impairments have been reported in patients after lesions to the right hemisphere (De Renzi and Nichelli, 1975; De Renzi et al., 1977; Hanley et al., 1991; Kessels et al., 2000; Postma et al., 2000), left PPC (Baldo and Dronkers, 2006), and right PPC (Husain et al., 2001; Malhotra et al., 2004, 2005, 2009; Pisella et al., 2004; Ravizza et al., 2005; Finke et al., 2006). As noted previously, earlier neuropsychological studies were unable to provide more detail regarding the anatomical boundaries of their patients' lesions due to a lack of brain imaging. A number of these studies tested spatial WM using the Corsi block-tapping task (Corsi, 1972) in which participants echo a sequence of taps across different locations as demonstrated by the experimenter (see Olson and Berryhill, 2009 for a summary of the spatial WM findings).

A few reports using other paradigms confirm a spatial WM deficit following right PPC damage. For example, in a population of unilateral right PPC patients with hemispatial neglect, WM deficits were observed across eye movements (Vuilleumier et al., 2007). The patient participants were impaired at remembering the spatial arrangement of stimuli in a spatial WM task where participants judged the location of a single target across a 2-3 s delay. When the task required eye movements during the delay period, a spatial WM deficit emerged only when gaze was redirected from left (neglected hemifield) to right (intact hemifield).

Other reports using different paradigms confirmed that WM could be impaired in patients with unilateral right PPC lesions who did not have neglect. In a series of tasks testing spatial, object and spatial-object conjunction WM, patients with right PPC lesions demonstrated a general WM deficit across spatial and non-spatial WM tasks (Berryhill and Olson, 2008b; but see Pisella et al., 2004). WM was tested using a recognition 
paradigm in which a probe image appeared after the maintenance period and participants judged whether the probe had appeared in the memory set or whether it was new. The manner by which WM was tested proved to be important. When object or order WM was tested using recognition bilateral PPC patients replicated the general WM deficit, but when WM was tested using recall, WM performance was normal (Berryhill and Olson, 2008a). This pattern of behavior extended beyond visual WM to aurally presented verbal stimuli (Berryhill et al., 2011). These findings seemed to point strongly toward PPC involvement in WM retrieval, since the WM deficits were associated with recognition rather than recall performance. However, these studies had used separate blocks of recall and recognition WM trials. The separation of tasks had been deliberate to keep the instructions simple. When recall and recognition trials were intermingled so that the retrieval demands were unpredictable, the bilateral PPC patients performed normally in both WM tasks (Berryhill et al., 2011). These data showed that the PPC damage did not eliminate the ability to make recognition WM judgments, but it did do something to patients' recognition performance when they knew they were going to be asked to make recognition judgments.

The distinction between recall and recognition WM performance may also guide the interpretation of several other findings in patients with bilateral PPC damage. First, two patients with bilateral PPC damage were able to accurately name the type of biological motion shown in point-light displays (Huberle et al., 2009). We also observed accurate identification of biological motion using point-light displays in EE555 (Berryhill and Olson, unpublished observations). This observation indicates that bilateral PPC damage does not entirely eliminate the ability to integrate multiple objects over time and argues against a general WM deficit. Since naming point-light displays requires recall responses, these findings are consistent with the successful performance on the recall WM tasks described above. Second, in a series of verbal whole report WM experiments, patient GK showed that he was able to recall more than a single item, but he required a significantly greater amount of time to perform WM tasks and to process information in general (Duncan et al., 2003). These authors interpreted their findings in terms of the Theory of Visual Attention (Bundesen, 1990; Bundesen et al., 2005, 2011) to suggest that the primary deficit was in processing speed. Although patients frequently require more time to process information it does not explain the difference in performance between recall and recognition WM trials. Finally, several papers have tested the importance of spatial factors in PPC patients' performance. The spatial separation between local elements in Navon letter stimuli modulates patients' performance such that they do better at identifying the global letter when the local items are closer together (Huberle and Karnath, 2006) or when the stimuli bias processing to intact parvocellular responses along the ventral visual stream (Thomas et al., 2012). Control participants perform similarly to bilateral PPC patients when their gaze was restricted (Dalrymple et al., 2010). These last findings provide added evidence that WM deficits after PPC lesions are subtle. This highlights the question of determining what the role of the PPC in WM might be.
One recent paper attempted to answer this question by examining WM deficits as a function of PPC subregion. Nature does not follow anatomical boundaries in lesion patients, which makes it difficult to parse anatomical distinctions. However, Koenigs et al. (2009) tested WM in a large number of patients with superior PPC or no superior PPC damage. They tested these populations on subcomponents of the Wechsler Memory scale (Wechsler, 1997). They found that damage to the SPL was impaired specifically when the tasks demanded manipulation rather than passive maintenance. For example, they found that WM performance was normal in the forward digit span task but abnormal in the backward digit span task (Koenigs et al., 2009). These findings serve as an important start in matching the more specific clusters of activity reported in fMRI findings with the disparate PPC damage in patient findings.

\section{ROLE OF THE PPC IN WM}

There are several hypothesized roles for PPC involvement in WM. The nature of these views is strongly shaped by the weighting of the data from the researcher's experimental approach. In other words, the starting point of the investigators influences the set of findings they emphasize. In a previous review, we described three proposals: information manipulation, information load and retrieval process (Olson and Berryhill, 2009). These views will be briefly noted below with the addition of a new proposal, the internal attention hypothesis.

\section{INFORMATION MANIPULATION}

One possibility is that the PPC, in particular the SPL is involved in manipulating information stored in WM. Several neuroimaging studies report increased superior parietal activations only when manipulation is required. Tasks requiring this kind of manipulation include spatially reordering stimuli based on cues (Wendelken, 2008), reordering the sequence of stimuli (Marshuetz et al., 2000; Wager and Smith, 2003; Marshuetz and Smith, 2006), or performing mental mathematical calculations (Dehaene et al., 1999, 2004). Additional support for this view comes from recent neuropsychological data showing impaired WM in patients with superior PPC damage only (Koenigs et al., 2009). The TMS literature provides complementary evidence with data showing that stimulation to superior PPC regions does not disrupt performance in a simple spatial WM task (recall or recognition), whereas TMS to the dorsolateral prefrontal cortex did impair WM (Hamidi et al., 2009). Several groups have reported that stimulating the superior PPC, particularly the right parietal lobe, improves reaction times in spatial WM tasks (Hamidi et al., 2008; Yamanaka et al., 2009).

One criticism of the information manipulation hypothesis is that PPC activations are observed in fMRI studies of WM tasks without manipulation demands (e.g., Todd and Marois, 2004, 2005; Xu and Chun, 2005, 2007; Schluppeck, 2006; Xu, 2007). This raises the question of the relationship between fMRI and other experimental techniques. A second criticism is that some sources of evidence seem to show TMS effects in WM tasks that do not require information manipulation, for example TMS speeding reaction times after SPL stimulation in simple WM 
tasks (Hamidi et al., 2008). However, it is important to note that depending on the paradigm, TMS may be facilitatory or inhibitory.

\section{PURE STORAGE AND RETRIEVAL PROCESS HYPOTHESES}

The finding that the WM retrieval task, recall or recognition, predicted intact, or impaired WM performance is inconsistent with the information load interpretation (Berryhill and Olson, 2008a; Berryhill et al., 2011; see also Berryhill et al., 2010b). This pattern was observed across several visual stimulus sets (tools, color patches, novel shapes, colorized Snodgrass drawings) (Berryhill and Olson, 2008a,b) and verbal stimuli (auditory words) (Berryhill et al., 2011). In each case the WM maintenance demands were the same, yet the performance differed. These data led us to propose PPC involvement in WM retrieval (Berryhill and Olson, 2008a,b; Olson and Berryhill, 2009). This explanation had the added benefit of fitting well with patients' selective deficits in episodic memory (Berryhill et al., 2007, 2011). This appealingly parsimonious explanation is no longer able to account for all of the neuropsychological findings and we have moved toward an internal attention account, described below.

\section{INTERNAL ATTENTION}

Unpredictable retrieval demands restore WM performance on recognition tasks in patients with bilateral PPC lesions (Berryhill et al., 2011). What explains the previous findings showing WM deficits for WM trials probed by recognition (Berryhill and Olson, 2008a; Berryhill et al., 2011)? We suspected that it had to do with differences in strategy when trials were intermingled rather than in separate blocks of recognition and recall WM trials. Accordingly, one role of the PPC, specifically bilateral IPL, is to maintain attention on items in WM to keep these representations from decaying. This process, sometimes referred to as attentional refreshing, refers to a recycling in and out of the attentional focus (Cowan, 1999; Chein, 2003; Barrouillet and Camos, 2009; Bledowski et al., 2009; Lewandowsky et al., 2009; Chein and Fiez, 2010). Importantly, this process is hypothesized to occur as a default material-general process, meaning it will update information currently in the focus of attention. When the focus of attention shifts to another item in WM items will begin to decay until the item returns to the focus. In addition there are active material-specific processes, such as the visuospatial buffer and phonological loop that maintain items in WM. Thus, during recall or interleaved blocks of trials, the material-specific maintenance mechanisms successfully support WM performance in patients with PPC damage presumably due to reliance on intact frontal structures. However, when there are blocks of recognition trials, PPC disruption caused by brain damage (Berryhill and Olson, 2008a,b; Berryhill et al., 2011) or tDCS (P4 stimulation) (Berryhill et al., 2010b) prevents successful attentional refreshing. Recent neuroimaging supports the view that strategy influences encoding related activations in frontoparietal regions at least in change detection tasks (Linke et al., 2011). Because participants have relied on this WM maintenance strategy and failed to supplement it with material-specific processes, their WM performance suffers.
This hypothesis makes the prediction that WM tasks requiring recall responses should not rely on the PPC whereas WM tasks requiring recognition responses should rely on PPC activity. There is some fMRI evidence supporting this prediction. The PPC is selectively activated during WM tasks probed by recognition but not during those probed by recall (Chein and Fiez, 2001, 2010; Chein et al., 2011). In these studies, Chein and colleagues are some of the few researchers to compare activity across different WM retrieval demands. Their data show that the SPL responds in a domain-general way such that it is strongly activated during verbal or spatial WM tasks during the encoding, maintenance and coordination phases. In short, the PPC is involved in a strategic manner during WM tasks. However, further work is needed to clarify the relative contributions of IPL and SPL regions to WM.

\section{CHALLENGES AND CONTINUATION}

The present review examined deficits in episodic memory and WM after PPC damage. Of course, it is important to remember that the neuropsychological approach has a series of limitations. As noted in the introduction, patient participants have lesions that damage multiple brain regions and subregions of the PPC. The lack of specificity in documenting structure-function associations is certain to be frustrating to researchers accustomed to other research methods such as fMRI. The cross-region lesion becomes more difficult to interpret as more functional subdivisions of the PPC are identified by newer techniques such as resting state and functional connectivity (Vincent et al., 2006, 2008; Nelson et al., 2010; Uddin et al., 2010; Zhang and Li, 2012) and dynamic causal modeling (Ma et al., 2011). These techniques also provide a new way of evaluating training regimes by evaluating changes in connectivity after training (Takeuchi et al., 2010). The resolution to differences between the fMRI and neuropsychological realm may be best addressed using neurostimulation techniques to temporarily lesion specific subregions of the larger PPC territory.

A second area where neuropsychology is silent is the phase or timecourse of PPC involvement. There are a number of experiments investigating PPC contributions during each phase(s) of memory (encoding, maintenance, or retrieval). Recent findings investigating episodic memory explore involvement during the phases of encoding (Wimber et al., 2010; Uncapher et al., 2011), maintenance (Buchsbaum et al., 2011), and retrieval (Xue et al., 2010; Seibert et al., 2011; Sestieri et al., 2011). Related findings exist for WM encoding (Tseng et al., 2010; Linke et al., 2011; Ravizza et al., 2011), maintenance (Todd and Marois, 2004; Xu and Chun, 2005; Ikkai and Curtis, 2011; Lepsien et al., 2011), and retrieval (Oztekin et al., 2009). Further advances from neurophysiology highlight additional factors such as the hemifield in determining WM capacity and fidelity (Buschman et al., 2011). Again, neurostimulation may be key to disentangling the temporal aspects of PPC involvement in various forms of memory while avoiding concerns related to cortical reorganization. Furthermore, patient participants may be in poor general health or on medications that affect cognition. Yet, in spite of these limitations, the neuropsychological approach is a powerful tool that raises provocative questions. Undoubtedly, recent and future 
findings using diffusion tensor imaging, functional connectivity, dynamic causal modeling, neurostimulation, even genetics, as well as fMRI and EEG, will prompt new questions to investigate in patient populations. In this sense it remains "early days" in understanding PPC contributions memory.

\section{REFERENCES}

Addis, D. R., Knapp, K., Roberts, R. P., and Schacter, D. L. (2012). Routes to the past: neural substrates of direct and generative autobiographical memory retrieval. Neuroimage 59, 2908-2922.

Addis, D., Wong, A., and Schacter, D. (2007). Remembering the past and imagining the future: common and distinct neural substrates during event construction and elaboration. Neuropsychologia 45, 1363-1377.

Ally, B. A., Simons, J. S., McKeever, J. D., Peers, P. V., and Budson, A. E. (2008). Parietal contributions to recollection: electrophysiological evidence from aging and patients with parietal lesions. Neuropsychologia 46, 1800-1812.

Arbib, M. A., Bonaiuto, J. B., Jacobs, S., and Frey, S. H. (2009). Tool use and the distalization of the end-effector. Psychol. Res. 73, 441-462.

Baddeley, A. (2000). The episodic buffer: a new component of working memory? Trends Cogn. Sci. 4, 417-423.

Baddeley, A., Allen, R., and VarghaKhadem, F. (2010). Is the hippocampus necessary for visual and verbal binding in working memory? Neuropsychologia 48, 1089-1095.

Baldo, J. V., and Dronkers, N. F. (2006). The role of inferior parietal and inferior frontal cortex in working memory. Neuropsychology 20, 529-538.

Balint, R. (1909). Seelenlahmung des "Schauens", optische Ataxie, raumliche Storung der Aufmerksamkeit. Monatsschr. Psychiatr. Neurologie 25, 51-81.

Barrouillet, P., and Camos, V. (2009). Interference: unique source of forgetting in working memory? Trends Cogn. Sci. 13, 145-146; author reply 146-147.

Berman, R., and Colby, C. (2009). Attention and active vision. Vision Res. 49, 1233-1248.

Berryhill, M. E., Chein, J. M., and Olson, I. R. (2011). At the intersection of attention and memory: the mechanistic role of the posterior parietal lobe in working memory. Neuropsycholgia 49, 1306-1315.

Berryhill, M. E., Drowos, D. B., and Olson, I. R. (2009). Bilateral parietal cortex damage does not impair associative memory for paired stimuli. Cogn. Neuropsychol. 26, 606-619.

Berryhill, M. E., and Olson, I. R. (2008a). Is the posterior parietal lobe involved in working memory retrieval? Evidence from patients with bilateral parietal lobe damage. Neuropsychologia 46, 1775-1786.

Berryhill, M. E., and Olson, I. R. (2008b). The right parietal lobe is critical for visual working memory. Neuropsychologia 46, 1767-1774.

Berryhill, M. E., Phuong, L., Picasso, L., Cabeza, R., and Olson, I. R. (2007). Parietal lobe and episodic memory: bilateral damage causes impaired free recall of autobiographical memory. J. Neurosci. 27, 14415-14423.

Berryhill, M. E., Picasso, L., Arnolds, R. A., Drowos, D. B., and Olson, I. R. (2010a). Similarities and differences between parietal and frontal patients in autobiographical and constructed experience tasks. Neuropsychologia 48, 1385-1393.

Berryhill, M. E., Wencil, E. B., Coslett, H. B., and Olson, I. R. (2010b). A selective working memory impairment after transcranial direct current stimulation to the right parietal lobe. Neurosci. Lett. 479, 312-316.

Berthoz, A. (1997). Parietal and hippocampal contribution to topokiPhilos. Trans. R. Soc. Lond. B Biol. Sci. 352, 1437-1448.

Bledowski, C., Rahm, B., and Rowe, J. B. (2009). What "works" in working memory? Separate systems for selection and updating of critical information. J. Neurosci. 29, 13735-13741.

Bucci, D. J. (2009). Posterior parietal cortex: an interface between attention and learning? Neurobiol. Learn. Mem. 91, 114-120.

Buchsbaum, B. R., Padmanabhan, A., and Berman, K. F. (2011). The neural substrates of recognition memory for verbal information: spanning the divide between shortand long-term memory. J. Cogn. Neurosci. 23, 978-991.

Buckner, R. L., and Carroll, D. C. (2007). Self-projection and the brain. Trends Cogn. Sci. 11, 49-57. netic and topographic memory.

\section{ACKNOWLEDGMENTS}

The University of Nevada supported this project through generous faculty start-up funding. I would like to thank Dr. Ingrid R. Olson for her continued mentoring. I also thank Dr. Gideon P. Caplovitz for his helpful comments.

Bundesen, C. (1990). A theory of visual attention. Psychol. Rev. 97, 523-547.

Bundesen, C., Habekost, T., and Kyllingsbaek, S. (2005). A neural theory of visual attention: bridging cognition and neurophysiology. Psychol. Rev. 112, 291-328.

Bundesen, C., Habekost, T., and Kyllingsbaek, S. (2011). A neural theory of visual attention and short-term memory (NTVA). Neuropsychologia 49, 1446-1457.

Burgess, P. W., Gonen-Yaacovi, G., and Volle, E. (2011). Functional neuroimaging studies of prospective memory: what have we learnt so far? Neuropsychologia 49, 2246-2257.

Burianova, H., McIntosh, A. R., and Grady, C. L. (2010). A common functional brain network for autobiographical, episodic, and semantic memory retrieval. Neuroimage 49, 865-874.

Buschman, T. J., Siegel, M., Roy, J. E., and Miller, E. K. (2011). Neural substrates of cognitive capacity limitations. Proc. Natl. Acad. Sci. U.S.A. 108, 11252-11255.

Butters, N., Samuels, I., Goodglass, H., and Brody, B. (1970). Short-term visual and auditory memory disorders after parietal and frontal lobe damage. Cortex 6, 440-459.

Cabeza, R. (2008). Role of lateral posterior parietal regions in episodic memory retrieval: the dual attention hypothesis. Neuropsychologia 46, 1813-1827.

Cabeza, R., Ciaramelli, E., Olson, I. R., and Moscovitch, M. (2008). The parietal cortex and episodic memory: an attentional account. Nat. Rev. Neurosci. 9, 613-625.

Cabeza, R., and St. Jacques, P. (2007). Functional neuroimaging of autobiographical memory. Trends Cogn. Sci. 11, 219-227.

Caspers, S., Schleicher, A., BachaTrams, M., Palomero-Gallagher, N., Amunts, K., and Zilles, K. (2012). Organization of the human inferior parietal lobule based on receptor architectonics. Cereb. Cortex. doi: 10.1093/cercor/bhs048. [Epub ahead of print].

Chafee, M. V., and Goldman-Rakic, P. S. (1998). Matching patterns of activity in primate prefrontal area $8 \mathrm{a}$ and parietal area 7ip neurons during a spatial working memory task. J. Neurophysiol. 79, 2919-2940.

Chambers, C. D., and Mattingley, J. B. (2005). Neurodisruption of selective attention: insights and implications. Trends Cogn. Sci. 9, 542-550.

Chein, J. (2003). Using neuroimaging to evaluate models of working memory and their implications for language processing. J. Neurolinguist. 16, 315-339.

Chein, J. M., and Fiez, J. A. (2001). Dissociation of verbal working memory system components using a delayed serial recall task. Cereb. Cortex 11, 1003-1014.

Chein, J. M., and Fiez, J. A. (2010). Evaluating models of working memory through the effects of concurrent irrelevant information. J. Exp. Psychol. Gen. 139, 117-137.

Chein, J. M., Moore, A. B., and Conway, A. R. (2011). Domaingeneral mechanisms of complex working memory span. Neuroimage 54, 550-559.

Ciaramelli, E., Grady, C., Levine, B., Ween, J., and Moscovitch, M. (2010). Top-down and bottom-up attention to memory are dissociated in posterior parietal cortex: neuroimagingand and neuropsychological evidence. J. Neurosci. 30, 4943-4956.

Constantinidis, C., and Procyk, E. (2004). The primate working memory networks. Cogn. Affect. Behav. Neurosci. 4, 444-465.

Corbetta, M., Patel, G., and Shulman, G. L. (2008). The reorienting system of the human brain: from environment to theory of mind. Neuron 58, 306-324.

Corbetta, M., and Shulman, G. L. (2002). Control of goal-directed and stimulus-driven attention in the brain. Nat. Rev. Neurosci. 3, 201-215.

Corbetta, M., and Shulman, G. L. (2011). Spatial neglect and attention networks. Annu. Rev. Neurosci. 34, 569-599.

Corsi, P. M. (1972). Human memory and the medial temporal region of the brain. Diss. Abstr. Int. 34, 819B.

Cowan, N. (1999). "An embeddedprocesses model of working memory," in Models of Working Memory: Mechanisms of Active 
Maintenance and Executive Control, eds A. Miyake and P. Shah (New York, NY: Cambridge University Press), 62-101.

Cowan, N. (2008). What are the differences between long-term, shortterm, and working memory? Prog. Brain Res. 169, 323-338.

Critchley, M. (1953). The Parietal Lobes. London, UK: Edward Arnold.

Culham, J. C., and Kanwisher, N. G. (2001). Neuroimaging of cognitive functions in human parietal cortex. Curr. Opin. Neurobiol. 11, 157-163.

Dalrymple, K. A., Bischof, W. F., Cameron, D., Barton, J. J., and Kingstone, A. (2010). Simulating simultanagnosia: spatially constricted vision mimics local capture and the global processing deficit. Exp. Brain Res. 202, 445-455.

Davidson, P. S., Anaki, D., Ciaramelli, E., Cohn, M., Kim, A. S., Murphy, K. J., Troyer, A. K., Moscovitch, M., and Levine, B. (2008). Does lateral parietal cortex support episodic memory? Evidence from focal lesion patients. Neuropsychologia 46, 1743-1755.

De Renzi, E., Faglioni, P., and Previdi, P. (1977). Spatial memory and hemispheric locus of lesion. Cortex 13, 424-433.

De Renzi, E., and Nichelli, P. (1975). Verbal and non-verbal short-term memory impairment following hemispheric damage. Cortex 11, 341-354.

Deese, J. (1959). On the prediction of occurrence of particular verbal intrusions in immediate recall. $J$. Exp. Psychol. 58, 17-22.

Dehaene, S., Molko, N., Cohen, L., and Wilson, A. J. (2004). Arithmetic and the brain. Curr. Opin. Neurobiol. 14, 218-224.

Dehaene, S., Spelke, E., Pinel, P., Stanescu, R., and Tsivkin, S. (1999). Sources of mathematical thinking: behavioral and brainimaging evidence. Science 284, 970-974.

Drew, T. W., McCollough, A. W., and Vogel, E. K. (2006). Event-related potential measures of visual working memory. Clin. EEG Neurosci. 37, 286-291.

Driver, J., Blankenburg, F., Bestmann, S., and Ruff, C. C. (2010). New approaches to the study of human brain networks underlying spatial attention and related processes. Exp. Brain Res. 206, 153-162.

Drowos, D., Berryhill, M. E., Andre, J., and Olson, I. R. (2010). True memory, false memory and the subjective recollection deficits after focal bilateral parietal lobe lesions. Neuropsychology 24, 465-475.
Duarte, A., Henson, R. N., and Graham, K. S. (2011). Stimulus content and the neural correlates of source memory. Brain Res. 1373, 110-123.

Duncan, J., Bundesen, C., Olson, A., Humphreys, G., Ward, R., Kyllingsbæk, S., van Raamsdonk, M., Rorden, C., and Chavda, S. (2003). Attentional functions in dorsal and ventral simultanagnosia. Cogn. Neuropsychol. 20, 675-701.

Ezzyat, Y., and Olson, I. R. (2008). The medial temporal lobe and visual working memory: comparisons across tasks, delays, and visual similarity. Cogn. Affect. Behav. Neurosci. 8, 32-40.

Finke, K., Bublak, P., and Zihl, J. (2006). Visual spatial and visual pattern working memory: neuropsychological evidence for a differential role of left and right dorsal visual brain. Neuropsychologia 44, 649-661.

Frey, S. H. (2008). Tool use, communicative gesture and cerebral asymmetries in the modern human brain. Philos. Trans. R. Soc. Lond. B Biol. Sci. 363, 1951-1957.

Giovanello, K. S., Keane, M. M., and Verfaellie, M. (2006). The contribution of familiarity to associative memory in amnesia. Neuropsychologia 44, 1859-1865.

Gläscher, J., Rudrauf, D., Colom, R., Paul, L. K., Tranel, D., Damasio, H., and Adolphs, R. (2010). Distributed neural system for general intelligence revealed by lesion mapping. Proc. Natl. Acad. Sci. U.S.A. 107, 4705-4709.

Glover, S. (2004). Separate visual representations in the planning and control of action. Behav. Brain Sci. 27, 3-24; discussion 24-78.

Graham, K. S., Barense, M. D., and Lee, A. C. (2010). Going beyond LTM in the MTL: a synthesis of neuropsychological and neuroimaging findings on the role of the medial temporal lobe in memory and perception. Neuropsychologia 48, 831-853.

Hamidi, M., Tononi, G., and Postle, B. (2008). Evaluating frontal and parietal contributions to spatial working memory with repetitive transcranial magnetic stimulation. Brain Res. 1230, 202-210.

Hamidi, M., Tononi, G., and Postle, B. (2009). Evaluating the role of prefrontal and parietal cortices in memory-guided response with repetitive transcranial magnetic stimulation. Neuropsychologia 47, 295-302.

Hanley, J. R., Young, A. W., and Pearson, N. A. (1991). Impairment of the visuo-spatial sketch pad. Q. J. Exp. Psychol. 43A, 101-125.

Hannula, D. E., Tranel, D., and Cohen, N. J. (2006). The long and the short of it: relational memory impairments in amnesia, even at short lags. J. Neurosci. 26, 8352-8359.

Haramati, S., Soroker, N., Dudai, Y., and Levy, D. A. (2008). The posterior parietal cortex in recognition memory: a neuropsychological study. Neuropsychologia 46, 1756-1766.

Hassabis, D., Kumaran, D., Vann, S. D., and Maguire, E. A. (2007). Patients with hippocampal amnesia cannot imagine new experiences. Proc. Natl. Acad. Sci. U.S.A. 104, 1726-1731.

Hassabis, D., and Maguire, E. A. (2007). Deconstructing episodic memory with construction. Trends Cogn. Sci. 11, 299-306.

Heilman, K. M., Watson, R. T., and Schulman, H. M. (1974). A unilateral memory defect. J. Neurol. Neurosurg. Psychiatry 37, 790-793.

Huberle, E., and Karnath, H. O. (2006). Global shape recognition is modulated by the spatial distance of local elements-evidence from simultanagnosia. Neuropsychologia 44, 905-911.

Huberle, E., Rupek, P., Lappe, M., and Karnath, H.-O. (2009). Perception of global gestalt by temporal integration in simultanagnosia. Eur. J. Neurosci. 29, 197-204.

Hunkin, N. M., Parkin, A. J., Bradley, V. A., Burrows, E. H., Aldrich, F. K., Jansari, A., and Burdon-Cooper, C. (1995). Focal retrograde amnesia following closed head injury: a case study and theoretical account. Neuropsychologia 33, 509-523.

Husain, M., Mannan, S., Hodgson, T., Wojciulik, E., Driver, J., and Kennard, C. (2001). Impaired spatial working memory across saccades contributes to abnormal search in parietal neglect. Brain 124, 941-952.

Husain, M., and Nachev, P. (2007). Space and the parietal cortex. Trends Cogn. Sci. 11, 30-36.

Hutchinson, J. B., Uncapher, M. R., and Wagner, A. D. (2009). Posterior parietal cortex and episodic retrieval: convergent and divergent effects of attention and memory. Learn. Mem. 16, 343-356.

Ikkai, A., and Curtis, C. E. (2011). Common neural mechanisms supporting spatial working memory, attention and motor intention. Neuropsychologia 49, 1428-1434.

Israel, L., and Schacter, D. L. (1997). Pictorial encoding reduces false recognition of semantic associates. Psychon. Bull. Rev. 4, 577-581.
Jax, S. A., and Coslett, H. B. (2009). Disorders of the perceptual-motor system. Adv. Exp. Med. Biol. 629, 377-391.

Jonides, J., Lewis, R. L., Nee, D. E., Lustig, C. A., Berman, M. G., and Moore, K. S. (2008). The mind and brain of short-term memory. Annu. Rev. Psychol. 59, 193-224.

Kahn, I., Davachi, L., and Wagner, A. D. (2004). Functional-neuroanatomic correlates of recollection: implications for models of recognition memory. J. Neurosci. 24, 4172-4180.

Kessels, R. P., van Zandvoort, M. J., Postma, A., Kappelle, L. J., and de Haan, E. H. (2000). The Corsi Block-Tapping Task: standardization and normative data. Appl. Neuropsychol. 7, 252-258.

Kim, H. (2011). Neural activity that predicts subsequent memory and forgetting: a meta-analysis of $74 \mathrm{fMRI}$ studies. Neuroimage 54, 2446-2461.

Kim, H., and Cabeza, R. (2007). Trusting our memories: dissociating the neural correlates of confidence in veridical versus illusory memories. J. Neurosci. 27, 12190-12197.

Kim, H., and Cabeza, R. (2009). Common and specific brain regions in high- versus low-confidence recognition memory. Brain Res. 1282, 103-113.

Koenigs, M., Barbey, A. K., Postle, B. R., and Grafman, J. (2009). Superior parietal cortex is critical for the manipulation of information in working memory. J. Neurosci. 29, 14980-14986.

Kondo, K., Maruishi, M., Ueno, H., Sawada, K., Hashimoto, Y., Ohshita, T., Takahashi, T., Ohtsuki, T., and Matsumoto, M. (2010). The pathophysiology of prospective memory failure after diffuse axonal injury-lesion-symptom analysis using diffusion tensor imaging. BMC Neurosci. 11, 147.

Kopelman, M. D., Wilson, B. A., and Baddeley, A. D. (1989). The autobiographical memory interview: a new assessment of autobiographical and personal semantic memory in amnesic patients. J. Clin. Exp. Neuropsychol. 11, 724-744.

Landis, T. (2000). Disruption of space perception due to cortical lesions. Spat. Vis. 13, 179-191.

Lepsien, J., Thornton, I., and Nobre, A C. (2011). Modulation of workingmemory maintenance by directed attention. Neuropsychologia 49, 1569-1577.

Levine, B. (2004). Autobiographical memory and the self in time: brain lesion effects, functional neuroanatomy, and lifespan development. Brain Cogn. 55, 54-68. 
Levine, B., Svoboda, E., Hay, J. F., Winocur, G., and Moscovitch, M. (2002). Aging and autobiographical memory: dissociating episodic from semantic retrieval. Psychol. Aging 17, 677-689.

Lewandowsky, S., Oberauer, K., and Brown, G. D. A. (2009). No temporal decay in verbal short-term memory. Trends Cogn. Sci. 13, 120-126.

Linke, A. C., Vicente-Grabovetsky, A., Mitchell, D. J., and Cusack, R. (2011). Encoding strategy accounts for individual differences in change detection measures of VSTM. Neuropsychologia 49, 1476-1486.

Ma, L., Steinberg, J. L., Hasan, K. M., Narayana, P. A., Kramer, L. A., and Moeller, F. G. (2011). Working memory load modulation of parieto-frontal connections: evidence from dynamic causal modeling. Hum. Brain Mapp. doi: 10.1002/ hbm.21329. [Epub ahead of print].

Maldonado, I. L., Moritz-Gasser, S., de Champfleur, N. M., Bertram, L., Moulinie, G., and Duffau, H. (2011). Surgery for gliomas involving the left inferior parietal lobule: new insights into the functional anatomy provided by stimulation mapping in awake patients. J. Neurosurg. 115, 770-779.

Malhotra, P., Coulthard, E. J., and Husain, M. (2009). Role of right posterior parietal cortex in maintaining attention to spatial locations over time. Brain 132, 645-660.

Malhotra, P., Jäger, H. R., Parton, A., Greenwood, R., Playford, E. D., Brown, M. M., Driver, J., and Husain, M. (2005). Spatial working memory capacity in unilateral neglect. Brain 128, 424-435.

Malhotra, P., Mannan, S., Driver, J., and Husain, M. (2004). Impaired spatial working memory: one component of the visual neglect syndrome? Cortex 40, 667-676.

Marshuetz, C., and Smith, E. E. (2006). Working memory for order information: multiple cognitive and neural mechanisms. Neuroscience 139, 195-200.

Marshuetz, C., Smith, E. E., Jonides, J., DeGutis, J., and Chenevert, T. L. (2000). Order information in working memory: fMRI evidence for parietal and prefrontal mechanisms. J. Cogn. Neurosci. 12(Suppl. 2), 130-144.

Milner, B. (1968). Visual recognition and recall after right temporal-lobe excision in man. Neuropsychologia 6, 191-209.

Nelson, S. M., Cohen, A. L., Power, J. D., Wig, G. S., Miezin, F. M., Wheeler, M. E., Velanova, K.,
Donaldson, D. I., Phillips, J. S., Schlaggar, B. L., and Petersen, S. E. (2010). A parcellation scheme for human left lateral parietal cortex. Neuron 67, 156-170.

Niendam, T. A., Laird, A. R., Ray, K. L., Dean, Y. M., Glahn, D. C., and Carter, C. S. (2012). Meta-analytic evidence for a superordinate cognitive control network subserving diverse executive functions. Cogn. Affect. Behav. Neurosci. 12, 241-268.

Olson, I. R., and Berryhill, M. (2009). Some surprising findings on the involvement of the parietal lobe in human memory. Neurobiol. Learn. Mem. 91, 155-165.

Olson, I. R., Moore, K. S., Stark, M., and Chatterjee, A. (2006a). Visual working memory is impaired when the medial temporal lobe is damaged. J. Cogn. Neurosci. 18, 1087-1097.

Olson, I. R., Page, K., Sledge, K., Chatterjee, A., and Verfaellie, M. (2006b). Working memory for conjunctions relies on the medial temporal lobe. J. Neurosci. 26, 4596-4601.

Oztekin, I., McElree, B., Staresina, B. P., and Davachi, L. (2009). Working memory retrieval: contributions of the left prefrontal cortex, the left posterior parietal cortex, and the hippocampus. J. Cogn. Neurosci. 21, 581-593.

Pisella, L., Berberovic, N., and Mattingley, J. B. (2004). Impaired working memory for location but not for colour or shape in visual neglect: a comparison of parietal and non-parietal lesions. Cortex 40, 379-390.

Pisella, L., Sergio, L., Blangero, A., Torchin, H., Vighetto, A., and Rossetti, Y. (2009). Optic ataxia and the function of the dorsal stream: contributions to perception and action. Neuropsychologia 47, 3033-3044.

Postma, A., Kessels, R. P., and van Asselen, M. (2008). How the brain remembers and forgets where things are: the neurocognition of object-location memory. Neurosci. Biobehav. Rev. 32, 1339-1345.

Postma, A., Sterken, Y., de Vries, L., and de Haan, E. H. F. (2000). Spatial localization in patients with unilateral posterior left or right hemisphere lesions. Exp. Brain Res. 134, 220-227.

Radvansky, G. A. (2005). Situation models, propositions, and the fan effect. Psychon. Bull. Rev. 12, 478-483.

Ranganath, C., Cohen, M. X., and Brozinsky, C. J. (2005). Working memory maintenance contributes to long-term memory formation: neural and behavioral evidence. $J$. Cogn. Neurosci. 17, 994-1010.

Ranganath, C., and D'Esposito, M. (2005). Directing the mind's eye: prefrontal, inferior, and medial temporal mechaqnisms for visual working memory. Curr. Opin. Neurobiol. 15, 175-182.

Ravizza, S. M., Behrmann, M., and Fiez, J. A. (2005). Right parietal contributions to verbal working memory: spatial or executive? Neuropsychologia 43, 2057-2067.

Ravizza, S. M., Hazeltine, E., Ruiz, S., and Zhu, D. C. (2011). Left TPJ activity in verbal working memory: implications for storageand sensory-specific models of short term memory. Neuroimage 55, 1836-1846.

Rawley, J. B., and Constantinidis, C. (2009). Neural correlates of learning and working memory in the primate posterior parietal cortex. Neurobiol. Learn. Mem. 91, 129-138.

Roediger, H. L. I., and McDermott, K. B. (1995). Creating false memories: remembering words not presented in lists. J. Exp. Psychol. Learn. Mem. Cogn. 21, 803-814.

Rugg, M., and Curran, T. (2007) Event-related potentials and recognition memory. Trends Cogn. Sci. 11, 251-257.

Ryan, J. D., and Cohen, N. J. (2004). Processing and short-term retention of relational information in amnesia. Neuropsychologia 42, 497-511.

Satoh, M., Nakase, T., Nagata, K., and Tomimoto, H. (2011). Musical anhedonia: selective loss of emotional experience in listening to music. Neurocase 17, 410-417.

Schluppeck, D. (2006). Sustained activity in topographic areas of human posterior parietal cortex during memory-guided saccades. J. Neurosci. 26, 5098-5108.

Schoo, L. A., van Zandvoort, M. J., Biessels, G. J., Kappelle, L. J., Postma, A., and de Haan, E. H. (2011). The posterior parietal paradox: why do functional magnetic resonance imaging and lesion studies on episodic memory produce conflicting results? J. Neuropsychol. 5, 15-38.

Scoville, W. B., and Milner, B. (1957). Loss of recent memory after bilateral hippocampal lesions. J. Neurosurg. Psychiatry 20, 11-21.

Seibert, T. M., Gimbel, S. I., Hagler, D. J. Jr., and Brewer, J. B. (2011) Parietal activity in episodic retrieval measured by fMRI and MEG. Neuroimage 55, 788-793.

Sestieri, C., Corbetta, M., Romani, G. L., and Shulman, G. L. (2011)
Episodic memory retrieval, parietal cortex, and the default mode network: functional and topographic analyses. J. Neurosci. 31, 4407-4420.

Shimamura, A. P. (2011). Episodic retrieval and the cortical binding of relational activity. Cogn. Affect. Behav. Neurosci. 11, 277-291.

Simons, J. S., Peers, P. V., Hwang, D. Y., Ally, B. A., Fletcher, P. C., and Budson, A. E. (2008). Is the parietal lobe necessary for recollection in humans? Neuropsychologia 46, 1185-1191.

Simons, J. S., Peers, P. V., Mazuz, Y. S., Berryhill, M. E., and Olson, I. R. (2010). Dissociation between memory accuracy and memory confidence following bilateral parietal lesions. Cereb. Cortex 20, 479-485.

St. Jacques, P. L., Kragel, P. A., and Rubin, D. C. (2011). Dynamic neural networks supporting memory retrieval. Neuroimage 57, 608-616.

Takeuchi, H., Sekiguchi, A., Taki, Y., Yokoyama, S., Yomogida, Y., Komuro, N., Yamanouchi, T., Suzuki, S., and Kawashima, R. (2010). Training of working memory impacts structural connectivity. J. Neurosci. 30, 3297-3303.

Thomas, C., Kveraga, K., Huberle, E., Karnath, H. O., and Bar, M. (2012). Enabling global processing in simultanagnosia by psychophysical biasing of visual pathways. Brain 135, 1578-1585.

Todd, J. J., and Marois, R. (2004). Capacity limit of visual short-term memory in human posterior parietal cortex. Nature 428, 751-754.

Todd, J. J., and Marois, R. (2005). Posterior parietal cortex activity predicts individual differences in visual short-term memory capacity. Cogn. Affect. Behav. Neurosci. 5, 144-155.

Tseng, P., Hsu, T. Y., Muggleton, N. G., Tzeng, O. J., Hung, D. L., and Juan, C. H. (2010). Posterior parietal cortex mediates encoding and maintenance processes in change blindness. Neuropsychologia 48, 1063-1070.

Tunik, E., Rice, N. J., Hamilton, A., and Grafton, S. T. (2007). Beyond grasping: representation of action in human anterior intraparietal sulcus. Neuroimage 36(Suppl. 2), T77-T86.

Uddin, L. Q., Supekar, K., Amin, H., Rykhlevskaia, E., Nguyen, D. A., Greicius, M. D., and Menon, V. (2010). Dissociable connectivity within human angular gyrus and intraparietal sulcus: evidence from functional and structura 
connectivity. Cereb. Cortex 20, 2636-2646.

Uncapher, M. R., Hutchinson, J. B., and Wagner, A. D. (2011). Dissociable effects of top-down and bottom-up attention during episodic encoding. J. Neurosci. 31, 12613-12628.

Uncapher, M. R., and Wagner, A. D. (2009). Posterior parietal cortex and episodic encoding: insights from fMRI subsequent memory effects and dual-attention theory. Neurobiol. Learn. Mem. 91, 139-154.

Vilberg, K. L., and Rugg, M. D. (2008). Memory retrieval and the parietal cortex: a review of evidence from a dual-process perspective. Neuropsychologia 46, 1787-1799.

Vincent, J. L., Kahn, I., Snyder, A. Z., Raichle, M. E., and Buckner, R. L. (2008). Evidence for a frontoparietal control system revealed by intrinsic functional connectivity. J. Neurophysiol. 100, 3328-3342.

Vincent, J. L., Snyder, A. Z., Fox, M. D., Shannon, B. J., Andrews, J. R., Raichle, M. E., and Buckner, R. L. (2006). Coherent spontaneous activity identifies a hippocampalparietal memory network. J. Neurophysiol. 96, 3517-3531.

Vuilleumier, P., Schwartz, S., Clarke, K., Husain, M., and Driver, J. (2002). Testing memory for unseen visual stimuli in patients with extinction and spatial neglect. J. Cogn. Neurosci. 14, 875-886.

Vuilleumier, P., Sergent, C., Schwartz, S., Valenza, N., Girardi, M., Husain, M., and Driver, J. (2007). Impaired perceptual memory of locations across gaze-shifts in patients with unilateral spatial neglect. J. Cogn. Neurosci. 19, 1388-1406.

Wager, T. D., and Smith, E. E. (2003). Neuroimaging studies of working memory: a meta-analysis. Cogn. Affect. Behav. Neurosci. 3, 255-274.

Wagner, A. D., Shannon, B. J., Kahn, I., and Buckner, R. L. (2005). Parietal lobe contributions to episodic memory retrieval. Trends Cogn. Sci. 9, 445-453.

Warrington, E. K., Logue, V., and Pratt, R. T. (1971). The anatomical localisation of selective impairment of auditory verbal shortterm memory. Neuropsychologia 9, 377-387.

Warrington, E. K., and Taylor, A. M. (1973). Immediate memory for faces: long- or short-term memory? Q. J. Exp. Psychol. 25, 316-322.

Wechsler, D. (1997). Wechsler Memory Scale-Third Edition: Administration and Scoring Manual. San Antonio, TX: Psychological Corporation.

Wendelken, C. (2008). Maintaining structured information: an investigation into functions of parietal and lateral prefrontal cortices. Neuropsychologia 46, 665-678.

Wheeler, M. E., and Buckner, R. L. (2004). Functional-anatomic correlates of remembering and knowing. Neuroimage 21, 1337-1349.

Wimber, M., Heinze, H. J., and Richardson-Klavehn, A. (2010). Distinct frontoparietal networks set the stage for later perceptual identification priming and episodic recognition memory. J. Neurosci. 30, 13272-13280.

Wojciulik, E., and Kanwisher, N. (1999). The generality of parietal involvement in visual attention. Neuron 23, 747-764.

$\mathrm{Xu}$, Y. (2007). The role of the superior intraparietal sulcus in supporting visual short-term memory for multifeature objects. J. Neurosci. 27, 11676-11686.

Xu, Y., and Chun, M. M. (2005). Dissociable neural mechanisms supporting visual short-term memory for objects. Nature 440, 91-95.

Xu, Y., and Chun, M. M. (2007). Visual grouping in human parietal cortex. Proc. Natl. Acad. Sci. U.S.A. 104, 18766-18771.

Xue, G., Dong, Q., Chen, C., Lu, Z. Mumford, J. A., and Poldrack, R. A. (2010). Greater neural pattern similarity across repetitions is associated with better memory. Science 330, 97-101.
Yamanaka, K., Yamagata, B., Tomioka, H., Kawasaki, S., and Mimura, M. (2009). Transcranial magnetic stimulation of the parietal cortex facilitates spatial working memory: near-infrared spectroscopy study. Cereb. Cortex 20, 1037-1045.

Zhang, S., and Li, C. S. (2012). Functional connectivity mapping of the human precuneus by resting state fMRI. Neuroimage 59 , 3548-3562.

Conflict of Interest Statement: The author declares that the research was conducted in the absence of any commercial or financial relationships that could be construed as a potential conflict of interest.

Received: 16 December 2011; accepted: 25 May 2012; published online: 11 June 2012.

Citation: Berryhill ME (2012) Insights from neuropsychology: pinpointing the role of the posterior parietal cortex in episodic and working memory. Front. Integr. Neurosci. 6:31. doi: 10.3389/fnint. 2012.00031

Copyright (C) 2012 Berryhill. This is an open-access article distributed under the terms of the Creative Commons Attribution Non Commercial License, which permits non-commercial use, distribution, and reproduction in other forums, provided the original authors and source are credited. 